\title{
Addendum to the MiniBooNE Run Plan: MiniBooNE Physics in 2006
}

November 2, 2004

A. A. Aguilar-Arevalo ${ }^{5}$, A. O. Bazarko ${ }^{12}$, C. Bhat ${ }^{7}$, S. J. Brice ${ }^{7}$, B. C. Brown ${ }^{7}$, L. Bugel ${ }^{5}$, J. Cao ${ }^{11}$, J. M. Conrad ${ }^{5}$, D. $\mathrm{Cox}^{8}$, A. Curioni $^{15}$, D. A. Finley ${ }^{7}$,

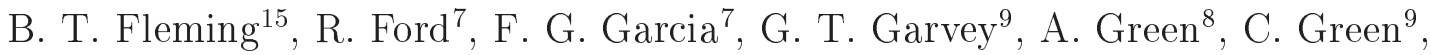
T. Hart ${ }^{4}$, E. Hawker ${ }^{14}$, R. Imlay ${ }^{10}$, R. A. Johnson ${ }^{3}$, P. Kasper ${ }^{7}$, T. Katori ${ }^{8}$, T. Kobilarcik ${ }^{7}$, I. Kourbanis ${ }^{7}$, S. Koutsoliotas ${ }^{2}$, J. M. Link ${ }^{5}$, Y. Liu ${ }^{1}$, Y. Liu ${ }^{11}$, W. C. Louis ${ }^{9}$, W. Marsh ${ }^{7}$, K. B. McConnel ${ }^{5}$, G. McGregor ${ }^{9}$, S. McKenney ${ }^{9}$, W. Metcalf ${ }^{10}$, H. O. Meyer ${ }^{8}$, P. D. Meyers ${ }^{12}$, G. B. Mills ${ }^{9}$, J. Monroe ${ }^{5}$, C. Moore ${ }^{7}$, R. H. Nelson ${ }^{4}$, P. Nienaber ${ }^{13}$, S. A. Ouedraogo ${ }^{10}$, R. B. Patterson ${ }^{12}$, E. Prebys ${ }^{7}$, J. L. Raaf ${ }^{3}$, H. Ray ${ }^{9}$, B. P. Roe ${ }^{11}$, A. D. Russell ${ }^{7}$, V. Sandberg ${ }^{9}$, B. Sapp ${ }^{9}$, R. Schirato ${ }^{9}$, D. Schmitz ${ }^{5}$, M. H. Shaevitz ${ }^{5}$, F. C. Shoemaker ${ }^{12}$, D. Smith ${ }^{6}$, M. Sorel ${ }^{5}$, P. Spentzouris ${ }^{7}$, I. Stancu ${ }^{1}$, R. Stefanski ${ }^{7}$, M. Sung ${ }^{10}$, H. A. Tanaka ${ }^{12}$, R. Tayloe ${ }^{8}$, R. Van de Water ${ }^{9}$, M. O. Wascko ${ }^{10}$, N. L. Walbridge ${ }^{9}$, D. H. White ${ }^{9}$, M. Wilking ${ }^{4}$, H. J. Yang ${ }^{11}$, G. P. Zeller ${ }^{5}$, E. D. Zimmerman ${ }^{4}$

\author{
${ }^{1}$ University of Alabama \\ ${ }^{2}$ Bucknell University \\ ${ }^{3}$ University of Cincinnati \\ ${ }^{4}$ University of Colorado \\ ${ }^{5}$ Columbia University \\ ${ }^{6}$ Embry Riddle Aeronautical University \\ ${ }^{7}$ Fermi National Accelerator Laboratory \\ ${ }^{8}$ Indiana University \\ ${ }^{9}$ Los Alamos National Laboratory \\ ${ }^{10}$ Louisiana State University \\ ${ }^{11}$ University of Michigan \\ ${ }^{12}$ Princeton University \\ ${ }^{13}$ St. Mary's University of Minnesota \\ ${ }^{14}$ Western Illinois University \\ ${ }^{15}$ Yale University
}




\section{Contents}

1 Executive Summary 2

$2 \quad \nu_{\mu} \rightarrow \nu_{e}$ Oscillation Search $\quad 4$

2.1 The Physics of LSND Oscillations . . . . . . . . . . . . . . . . 4

2.1.1 Physics of CP Violating Models . . . . . . . . . . . . . . 4

2.2 Three Possible Scenarios for a Result . . . . . . . . . . . . . . 5

2.2.1 Strong Signal . . . . . . . . . . . . . . . . . 6

2.2 .2 Inconclusive Result . . . . . . . . . . . . . . . . 6

2.2.3 LSND Excluded in Neutrino Mode . . . . . . . . . . . . . 6

3 Antineutrino Running in $2006 \quad 8$

3.1 Antineutrino Cross Section Measurements . . . . . . . . . . . . 8

3.1.1 Expected $\bar{\nu}_{\mu}$ Event Rates at MiniBooNE . . . . . . . . . . . 9

3.1.2 Wrong Sign Constraints ... . . . . . . . . . . . 10

3.1.3 CC Quasi-Elastic Scattering $\left(\bar{\nu}_{\mu} p \rightarrow \mu^{+} n\right) \ldots . . . . . .13$

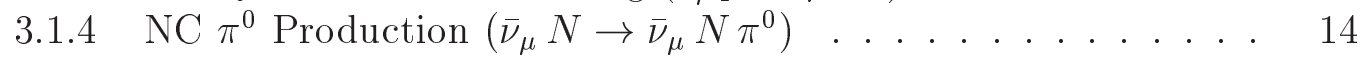

3.1.5 CC Resonant Single $\pi^{-}$Production $\left(\bar{\nu}_{\mu} N \rightarrow \mu^{+} N \pi^{-}\right) \ldots . .16$

3.2 Oscillation Searches . . . . . . . . . . . . . . . . . 17

$3.2 .1 \bar{\nu}_{\mu}$ Disappearance . . . . . . . . . . . . . . . . 17

$3.2 .2 \bar{\nu}_{e}$ Appearance . . . . . . . . . . . . . . . 18

4 Conclusions $\quad 20$ 


\section{Chapter 1}

\section{Executive Summary}

The primary physics goal of MiniBooNE is to confirm or rule out the LSND $\bar{\nu}_{\mu} \rightarrow \bar{\nu}_{e}$ oscillation observation with high significance. In the 2003 Run Plan document [1] presented to the PAC, we outlined the need for $1 \times 10^{21}$ protons on target (POT) to achieve this goal. In this document, we present an addendum to our run plan for fiscal year 2006 (FY2006).

The $\nu_{\mu} \rightarrow \nu_{e}$ appearance analysis is currently in progress. We anticipate having a result in the autumn of 2005 using data taken through the fiscal year 2005 run. Based on this result, we will decide how to proceed. There are three possible scenarios: strong confirmation, strong exclusion, or an inconclusive result [1]. There are two possible modes for continued running: the first is to continue running in neutrino mode, and the second option is to change the polarity of the magnetic horn and run in antineutrino mode. We will decide between these modes depending on the outcome of our $\nu_{e}$ analysis.

If the result is inconclusive, the FY2006 run would continue in neutrino mode with the goal of a definitive result as discussed in the Run Plan document [1]. If MiniBooNE strongly confirms LSND, the next step would be an accurate measurement of the oscillation parameters, which will ultimately be achieved with the addition of one or more detectors (BooNE). While planning for BooNE, we may continue to run in neutrino mode, as MiniBooNE is expected to be statistics limited up to $2 \times 10^{21}$ POT, or we may switch the polarity of the horn and run in antineutrino mode. In the case of a strong exclusion of the LSND oscillation in neutrino mode, we would choose to begin an antineutrino run in FY2006. As this document will show, one year of antineutrino running will produce significant new physics results, including a $\bar{\nu}_{\mu}$ disappearance oscillation search and the world's only $\bar{\nu}_{\mu}$ cross section measurements in this energy region. The sensitivity of the $\bar{\nu}_{\mu}$ disappearance oscillation analysis extends an order of magnitude lower than the current bound of $\Delta m^{2} \sim 10 \mathrm{eV}^{2}$. Cross section measurements of $\bar{\nu}_{\mu}$ charged current quasi-elastic scattering and single pion production, where current uncertainties are greater than $30 \%$, can be made with bet-

ter than $10 \%$ statistical precision. These results will leverage analyses performed in neutrino mode to constrain backgrounds and contamination from $\nu_{\mu}$-induced events. This year of antineutrino running may also serve as the first installment of a longer 
run that would complete the process of checking the LSND result, which was seen in antineutrino interactions. These physics goals were identified as strong priorities by the recent APS Interdivisional Neutrino Study [2], reflecting the broad interest of the neutrino community.

This extension to the MiniBooNE Run Plan is written in response to the Director's recent communication. In his letter, "Prospects for the Booster Neutrino Beam," dated August 6, 2004, the statement was made:

Collaborations proposing experiments to run in the Booster neutrino beam in FY2006 and beyond should plan their physics program on the basis of $1-2 \times 10^{20}$ POT per year. Proponents may want to discuss what additional physics could be done with somewhat more protons, but they should understand that is beyond our present expectations for the beam.

On the basis of this letter and the compelling physics case presented in this document, we ask the PAC to endorse that:

- $1-2 \times 10^{20}$ POT be delivered in FY2006 to the Booster neutrino beam line; and

- higher proton intensity should continue to be vigorously pursued.

We ask the PAC to endorse our strong physics program for FY2006. As per the request of the Director, we have also outlined the beginning of a multi-year program which we plan to present in the future. 


\section{Chapter 2}

\section{$\nu_{\mu} \rightarrow \nu_{e}$ Oscillation Search}

\subsection{The Physics of LSND Oscillations}

The physics motivation for MiniBooNE and a discussion of the theoretical ramifications of LSND oscillations are given in the MiniBooNE Run Plan [1]. One major theoretical insight that has come to light since the Run Plan is the role of CP violation in sterile neutrino models with two or more additional neutrinos.

\subsubsection{Physics of CP Violating Models}

If the LSND signal is due to oscillations, among the best models to explain all data are those which incorporate sterile neutrinos. Models with two or more additional sterile neutrinos (so-called $3+\mathrm{n}$ models) provide a good fit to the data [3] without necessarily violating cosmological bounds.

If more than one $\Delta m^{2}$ contributes to the oscillations, MiniBooNE can be sensitive to a $\mathrm{CP}$ violating $(\mathrm{CPV})$ phase in the mixing matrix. Only appearance measurements are sensitive to $\mathrm{CPV}$. This is the motivation for $\mathrm{CPV}$ searches at $\mathrm{NO} \nu \mathrm{A}$ and $\mathrm{T} 2 \mathrm{~K}$, where only the three active neutrinos are generally assumed. In the case of LSND-like oscillations, additional CPV phases may affect the CPV searches both at long and short neutrino oscillation baselines. For example, in 3+2 models [4], CPV phases can affect neutrino oscillations in short-baseline experiments, where the oscillation probability is given by:

$$
\begin{aligned}
P\left({ }_{\nu}^{(-)} \rightarrow \nu_{\mu}^{(-)}\right)= & 4\left|U_{e 4}\right|^{2}\left|U_{\mu 4}\right|^{2} \sin ^{2} x_{41}+4\left|U_{e 5}\right|^{2}\left|U_{\mu 5}\right|^{2} \sin ^{2} x_{51}+ \\
& 8\left|U_{e 4}\right|\left|U_{\mu 4}\right|\left|U_{e 5}\right|\left|U_{\mu 5}\right| \sin x_{41} \sin x_{51} \cos \left(x_{54} \mp \phi_{54}\right)
\end{aligned}
$$

where

$$
x_{j i} \equiv 1.27 \Delta m_{j i}^{2} L / E \quad \text { and } \quad \phi_{54} \equiv \arg \left(U_{e 4}^{*} U_{\mu 4} U_{e 5} U_{\mu 5}^{*}\right) .
$$

CPV affects the oscillation probability through the interference term appearing in the second line of this equation.

As a consequence of this model, expectations for MiniBooNE neutrino and antineutrino running can be significantly different. Figure 2.1 illustrates this result for 


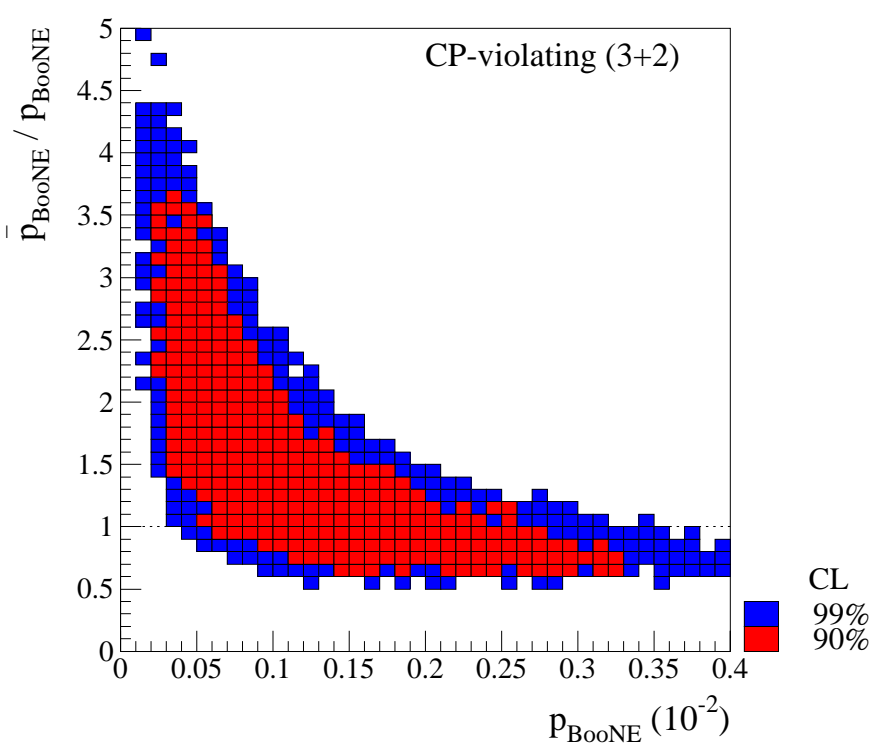

Figure 2.1: For MiniBooNE, the ratio of oscillation probabilities in $\bar{\nu}$ and $\nu$ modes as a function of the oscillation probability in $\nu$ mode given $3+2$ models allowed by existing data [4]. $\bar{P}_{B o o N E}$ and $P_{B o o N E}$ represent the combined flux and cross-section weighted oscillation probabilities for all $\nu$ 's and $\bar{\nu}$ 's in, respectively, $\bar{\nu}$ and $\nu$ running modes.

a sampling of $3+2$ models with CPV. For each point, there exists at least one combination of masses, mixings, and CPV phase which is consistent with the existing oscillation data, including LSND, at the indicated confidence level. The calculation uses the MiniBooNE neutrino and antineutrino mode fluxes (including $\nu_{e}$ and $\bar{\nu}_{e}$ backgrounds), thereby taking the energy dependence and "wrong-sign" 1 neutrino contamination into account. The abscissa is the MiniBooNE oscillation probability in neutrino mode and the ordinate is the ratio of the oscillation probability in antineutrino to neutrino mode. One can see that $\mathrm{CP}$ violation may enhance or decrease the oscillation probability in antineutrino mode with respect to neutrino mode, depending on the mixing parameters. For small values of oscillation probability in neutrino mode, CPV generally causes an enhancement (of up to a factor 4) in antineutrino mode.

\subsection{Three Possible Scenarios for a Result}

We anticipate having a $\nu_{e}$ appearance result before FY2006 running begins, assuming that the analysis has sufficiently matured. With this result, there are three possible outcomes: 1) a strong signal confirming LSND, 2) an inconclusive result, or 3) a definitive rejection of the hypothesis of LSND-like oscillations in neutrinos. While the objectives of an extended run may differ in each case, the potential for significant scientific output will strongly motivate continued running regardless of the $\nu_{e}$ appearance outcome.

\footnotetext{
${ }^{1}$ Throughout this document, wrong-sign content refers to neutrinos in an antineutrino beam, or antineutrinos in a neutrino beam.
} 


\subsubsection{Strong Signal}

If MiniBooNE sees a signal, it will be an indication that something interesting and unexpected is occurring in the neutrino sector. It will firmly establish that neutrinos oscillate at three distinct $\Delta m^{2}$ scales. This can only result from new physics such as the addition of one or more sterile neutrinos.

Having established the oscillation with a beam composed primarily of muon neutrinos, the collaboration will work to push this line of research forward on at least two fronts. First, we will begin planning for the construction of additional detectors at different baselines. The number of additional detectors and their baselines will be contingent on the precision and value of our mixing parameter measurements. Second, we will continue running with the existing MiniBooNE detector. Further neutrino running may be needed to better determine the value of the mixing parameters. Alternatively, if we have exhausted the potential of our neutrino data, we will switch the polarity of the horn and take antineutrino data. The objectives of an antineutrino program are several fold. First, a measurement of the oscillation with an antineutrino beam would have different systematic errors and could, therefore, strengthen the overall case for oscillations. Second, because MiniBooNE is an appearance measurement, the combination of oscillation results in neutrinos and antineutrinos may have sensitivity to CP violation as was discussed in Section 2.1.1. Finally, even a single year of antineutrino data would significantly improve the state of low energy antineutrino cross sections. The prospects for antineutrino cross sections are discussed in detail in Section 3.1.

\subsubsection{Inconclusive Result}

As discussed in detail in the MiniBooNE Run Plan [1], with less than $1 \times 10^{21} \mathrm{POT}$, an unfavorable statistical fluctuation in the rates of either signal or background events could shift what would otherwise be a definitive signal (or limit) into an inconclusive result. In this case, the obvious prescription will be to continue accumulating statistics in neutrino mode.

Under certain circumstances, it may be beneficial to reduce the beam decay length by inserting the 25 meter absorber. This configuration change may help address systematic uncertainties due to intrinsic $\nu_{e}$ contamination in the beam.

\subsubsection{LSND Excluded in Neutrino Mode}

In the case of a definitive exclusion of an LSND-like oscillation in neutrinos, it is essential to search for oscillations in antineutrinos, because, as was shown in Section 2.1.1, a small (even unmeasurable) oscillation rate in neutrinos does not preclude a sizable rate in antineutrinos. This program has been recognized by the recent APS Interdivisional Neutrino Study [2] as essential to resolving the question of LSND oscillations.

Our intention in this scenario is antineutrino running in FY2006 to be the first year in a multi-year $\bar{\nu}_{\mu} \rightarrow \bar{\nu}_{e}$ oscillation search. In addition to a $\bar{\nu}_{e}$ appearance search, there exists strong motivation for exploring $\bar{\nu}_{\mu}$ disappearance as well as a an important program of antineutrino cross section measurements, both of which can be addressed 
in the first year of running. The current knowledge of antineutrino cross sections in the range of $500 \mathrm{MeV}$ to $1.5 \mathrm{GeV}$ is poor, and even a single year of MiniBooNE data at $2 \times 10^{20}$ POT could yield significant advancement. The physics potential of $\bar{\nu}_{\mu}$ cross sections measurements, $\bar{\nu}_{\mu}$ disappearance, and $\bar{\nu}_{e}$ appearance are discussed in detail in the next chapter. 


\section{Chapter 3}

\section{Antineutrino Running in 2006}

Whether MiniBooNE confirms or rules out the LSND anomaly, it is desirable for MiniBooNE to run with antineutrinos. Since the LSND result was observed with antineutrinos, it is imperative to explore the LSND signal region using antineutrinos. This would include both $\bar{\nu}_{\mu}$ disappearance and $\bar{\nu}_{e}$ appearance searches. The MiniBooNE beam line is also a unique tool for studying antineutrino cross sections at low energy. These studies probe previously unexplored regions, thus providing key information for future programs such as $\mathrm{NO} \nu \mathrm{A}$ and $\mathrm{T} 2 \mathrm{~K}$. These possible cross section measurements and oscillation searches are discussed in the following two sections.

\subsection{Antineutrino Cross Section Measurements}

The APS Interdivisional Neutrino Study explicitly supports the:

Determination of the neutrino reaction and production cross sections required for a precise understanding of neutrino oscillation physics ...

and recommends that the search for $\mathrm{CP}$ violation in neutrino oscillations be given high priority [2]. Future explorations will most likely attempt to measure CP violation by comparing oscillation probabilities for $\nu_{\mu} \rightarrow \nu_{e}$ versus $\bar{\nu}_{\mu} \rightarrow \bar{\nu}_{e}$. To accomplish this, one needs control of the ratio of $\nu / \bar{\nu}$ cross sections to a better precision than the size of the expected asymmetry. In addition, if such measurements are to be made using a nuclear target to maximize event statistics, the antineutrino cross sections cannot simply be inferred from the neutrino cross sections since the nuclear effects for neutrino and antineutrino interactions are not identical. Figure 3.1 compares the size of the expected nuclear effects for neutrino and antineutrino quasi-elastic (QE) interactions on carbon. As a result of a softer $Q^{2}$ distribution, the antineutrino cross section reduction is roughly $20-30 \%$ larger than for neutrino scattering.

While published low energy neutrino cross section data are hardly copious [7], measurements of low energy antineutrino cross sections are even more scarce. Additional antineutrino data on nuclear targets are clearly needed so that interaction spectra and background rates for antineutrino oscillation experiments (including our own) can be estimated with confidence. Fermilab, through MiniBooNE, can be the

first to provide these necessary inputs with no additional detector cost and with the anticipated Booster proton delivery in FY2006. 


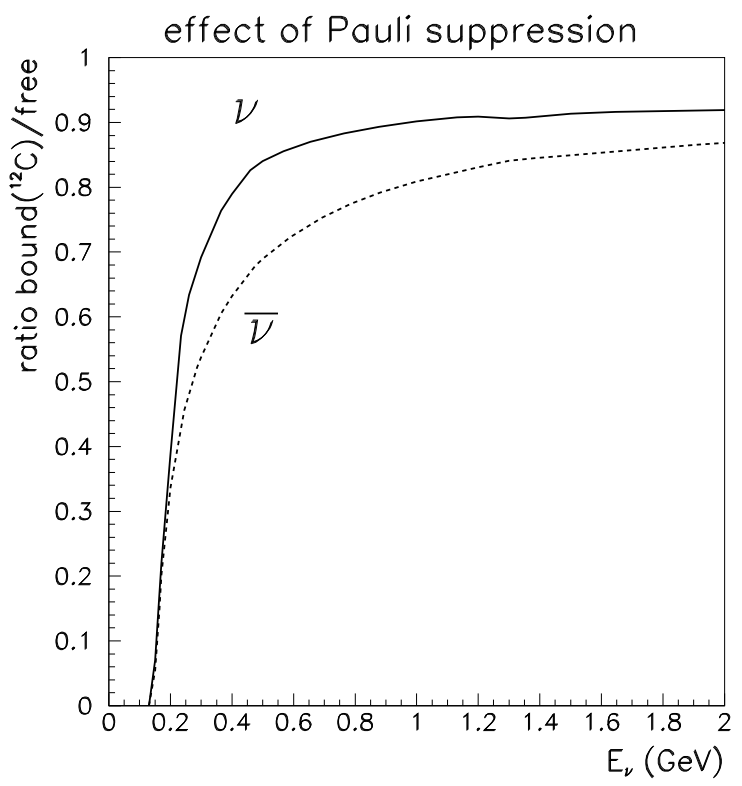

Figure 3.1: Size of the estimated nuclear effects for $\nu$ (solid) and $\bar{\nu}$ (dashed) $C C Q E$ interactions on carbon as a function of neutrino energy. Predictions are from the NUANCE Monte Carlo generator [6].

\subsubsection{Expected $\bar{\nu}_{\mu}$ Event Rates at MiniBooNE}

Historically, antineutrino reactions have been more challenging to measure than neutrino reactions because accelerator-based antineutrino experiments have suffered from lower fluxes, lower cross sections, and thus lower overall event rates. Typically, the fluxes and cross sections are each halved, leading to an overall factor of four reduction in antineutrino event rates. Antineutrino beams also have larger wrong-sign contamination than neutrino beams. In the MiniBooNE beam, antineutrinos are roughly $2 \%$ of events in neutrino running, whereas neutrinos constitute $\sim 30 \%$ of events in antineutrino running.

Despite the reduction in rate inherent in this and any other antineutrino beam, in just one year of running MiniBooNE expects antineutrino event statistics large enough to yield interesting and useful cross section measurements. We expect to use the same techniques to obtain a precise measure of our incoming flux in this mode as described in the MiniBooNE Run Plan [1]. Table 3.1 lists the expected antineutrino event statistics for $2 \times 10^{20}$ POT at MiniBooNE. As can be seen from this table, the most abundant interactions at MiniBooNE include charged current quasi-elastic (CC QE), neutral current elastic (NC EL), resonant single-pion production, and coherent pion production processes. Rates are listed for both right-sign (antineutrino) and wrongsign (neutrino) interactions. Note that wrong-sign interactions cannot be neglected in antineutrino running, as they can in neutrino running.

Given these projected statistics, this section describes potential cross section measurements that can be performed at MiniBooNE. First, we describe an innovative approach for measuring the wrong-sign contamination in the antineutrino data, followed by measurements of antineutrino $\mathrm{CC} \mathrm{QE}, \mathrm{NC} \pi^{0}$, and $\mathrm{CC}$ single-pion cross sections. 


\begin{tabular}{|l|r|r||r|}
\hline Reaction & $\bar{\nu}_{\mu}(\mathrm{RS})$ events & $\nu_{\mu}(\mathrm{WS})$ events & total $\left(\bar{\nu}_{\mu}+\nu_{\mu}\right)$ \\
\hline \hline CC QE & 32,476 & 11,234 & 43,709 \\
\hline NC elastic & 13,329 & 4,653 & 17,982 \\
\hline CC resonant $1 \pi^{-}$ & 7,413 & 0 & 7,413 \\
\hline CC resonant $1 \pi^{+}$ & 0 & 6,998 & 6,998 \\
\hline CC resonant $1 \pi^{0}$ & 2,329 & 1,380 & 3,709 \\
\hline NC resonant $1 \pi^{0}$ & 3,781 & 1,758 & 5,539 \\
\hline NC resonant $1 \pi^{+}$ & 1,414 & 654 & 2,067 \\
\hline NC resonant $1 \pi^{-}$ & 1,012 & 520 & 1,532 \\
\hline NC coherent $1 \pi^{0}$ & 2,718 & 438 & 3,156 \\
\hline CC coherent $1 \pi^{-}$ & 4,487 & 0 & 4,487 \\
\hline CC coherent $1 \pi^{+}$ & 0 & 748 & 748 \\
\hline other $($ multi- $\pi$, DIS $)$ & 2,589 & 2,156 & 4,745 \\
\hline \hline total & 71,547 & 30,539 & 102,086 \\
\hline
\end{tabular}

Table 3.1: Event rates expected in MiniBooNE $\bar{\nu}$ running with $2 \times 10^{20}$ POT assuming a $550 \mathrm{~cm}$ fiducial volume, before cuts. Listed are the expected right-sign (RS) and wrong-sign (WS) events for each reaction channel. These event estimates do not include the effects of final state interactions in carbon which can alter the composition of the observed final state, and do not include effects fom reconstruction.

\subsubsection{Wrong Sign Constraints}

Antineutrino cross section measurements, using conventional beams, must always confront a non-negligible neutrino contamination and MiniBooNE is no exception. To avoid sole reliance on beam Monte Carlo predictions, such backgrounds are commonly eliminated (at least in the case of CC interactions) by identifying the charge of the outgoing muon. Without a magnetic field to provide such event-by-event identification, MiniBooNE has developed several novel techniques for measuring wrong-sign backgrounds in the antineutrino data, allowing us to make more precise antineutrino cross section measurements. The wrong-sign content is constrained by three measurements: muon angular distributions in QE events, muon lifetimes, and CC single $\pi^{+}$events. The following subsections describe each of these independent approaches.

\section{Muon Angular Distributions}

The most powerful wrong-sign constraint comes from the observed direction of outgoing muons in CC QE interactions. As can be seen from Figure 3.2, neutrino and antineutrino events exhibit distinct muon angular distributions. The final state muons in antineutrino QE interactions predominantly follow the initial neutrino direction - they are more forward peaked than muons from neutrino interactions. This same phenomenon is observed in deep inelastic scattering (DIS), in which CC antineutrinoantiquark scattering is forbidden in the backwards direction. In hadronic terms, the suppression of antineutrino QE scattering at backward angles arises from the destructive interference between the $V A$ and $V V+A A$ terms in the cross section. This interference is constructive in the case of neutrino QE scattering. The excellent an- 

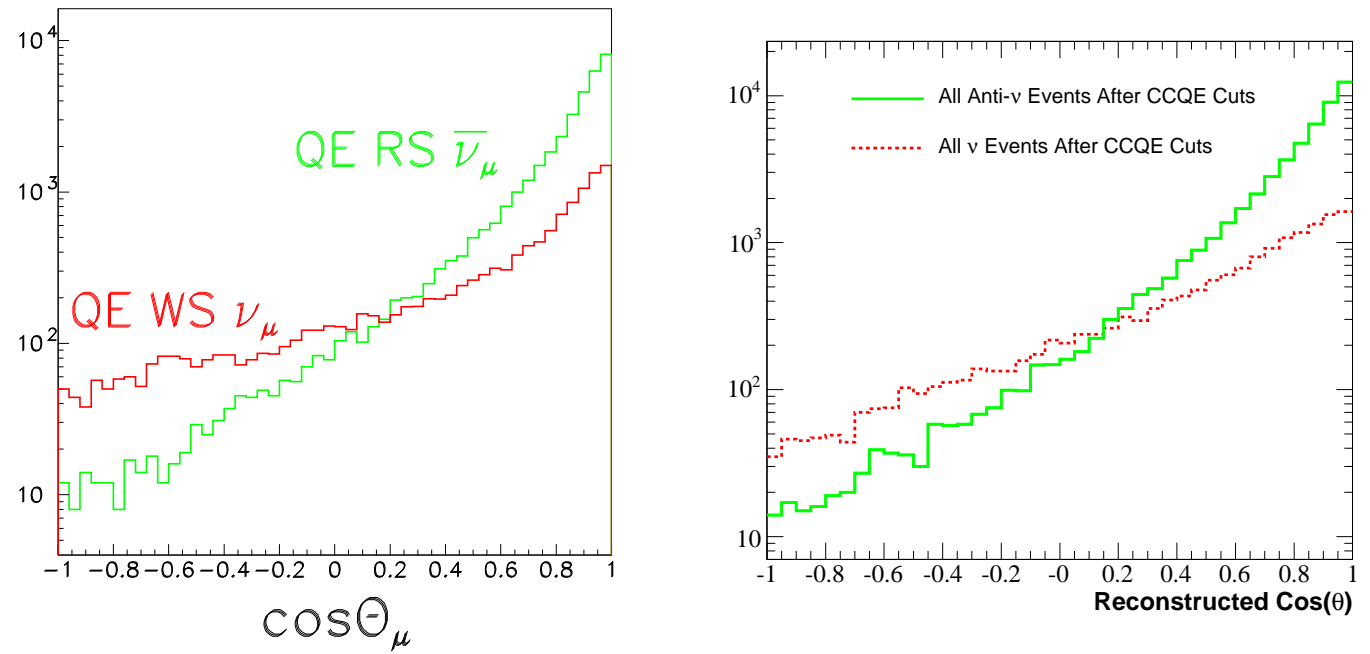

Figure 3.2: Generated (left) and reconstructed (right) muon angular distributions for $C C Q E$ right-sign $\bar{\nu}_{\mu}$ and wrong-sign $\nu_{\mu}$ interactions in antineutrino running at MiniBooNE. As in Table 3.1, neutrinos are predicted to comprise $\sim 30 \%$ of the events. Unlike the lefthand plot, the righthand plot includes non-QE backgrounds. The high degree of angular separation persists even after the events have been reconstructed and have passed $C C Q E$ event selection.

gular resolution in MiniBooNE allows us to exploit this difference and fit the angular distributions to extract the wrong-sign contribution.

Using a detailed Monte Carlo study, we find that the wrong-sign component can be reliably measured to $7 \%$ of itself after including systematic uncertainties and backgrounds. Using generated distributions, we find that the uncertainty is $5 \%$, indicating that the resolution of the event reconstruction and background uncertainties do not significantly affect the measurement. We have also studied fits to $Q^{2}$ distributions and achieved similar results.

\section{Muon Lifetimes}

A second handle results from measuring the rate at which muons decay in the MiniBooNE detector. Due to an $8 \% \mu^{-}$capture probability in mineral oil, positively and negatively charged muons exhibit different effective lifetimes $\left(\tau \sim 2.026 \mu \mathrm{s}\right.$ for $\mu^{-}[8]$ and $\tau \sim 2.197 \mu s$ for $\left.\mu^{+}[9]\right)$. Solely using this difference, we find that the wrongsign contribution can be extracted with a $30 \%$ statistical uncertainty and negligible systematic uncertainties. While not as precise as fits to the muon angular distributions, this particular constraint is unique, as it is independent of kinematics and reconstruction.

\section{Single $\pi^{+}$Event Sample}

A third measure makes use of the fact that $\mathrm{CC} 1 \pi^{+}$events in antineutrino mode almost exclusively result from neutrino interactions in the detector (Table 3.1). The 
dominant $\mathrm{CC}$ single pion production channels contain a final state $\pi^{+}$in the case of neutrino scattering, as opposed to a final state $\pi^{-}$in the case of antineutrino scattering.

MiniBooNE cleanly identifies CC $1 \pi^{+}$events by locating the two decay electrons that follow the primary neutrino interaction, one each from the $\mu^{-}$and $\pi^{+}$decay chains [10]. CC $1 \pi^{-}$events are largely rejected by this requirement because most of the $\pi^{-}$'s come to rest and are captured by carbon nuclei, resulting in no decay electrons. There is a residual background from $\mathrm{CC} 1 \pi^{0}$ events in which the final state pion charge exchanges $\left(\pi^{0} \rightarrow \pi^{+}\right)$, but this background is estimated to be small, around $2 \%$. The probability for double charge exchange $\pi^{-} \rightarrow \pi^{+}$is highly suppressed, and can be completely neglected. Starting from a sample that is $70 \%$ right-sign antineutrino interactions, this simple two decay electron requirement yields an $85 \%$ pure sample of wrong-sign neutrino events (Table 3.2).

\begin{tabular}{|c||c|c|}
\hline Neutrino type & \# before cuts & \# after CC $\pi^{+}$event selection \\
\hline$\nu_{\mu}(\mathrm{WS})$ & 30,539 & 2,525 \\
\hline $\bar{\nu}_{\mu}(\mathrm{RS})$ & 71,547 & 461 \\
\hline \hline total & 102,086 & 2,986 \\
\hline
\end{tabular}

Table 3.2: Total number of generated events compared to the number of reconstructed events passing the $C C 1 \pi^{+}$selection in antineutrino running assuming $2 \times 10^{20}$ POT.

Assigning very conservative uncertainties to the antineutrino background events and the $\mathrm{CC} 1 \pi^{+}$cross section, which will be well-measured by the MiniBooNE neutrino data, yields a $15 \%$ uncertainty on the wrong-sign content in the beam given $2 \times 10^{20}$ POT. Increased statistics yield only marginal improvement in this measurement. Gains could be made by attempting to reduce the antineutrino backgrounds in this sample; however, no such attempt to optimize the event selection was made for this initial study. This measurement is complementary to the muon angular distribution determination because $\mathrm{CC} 1 \pi^{+}$events predominantly result from resonance decays, and therefore constrain the wrong-sign content at larger neutrino energies.

\section{Summary of Wrong-Sign Measurements in the $\bar{\nu}$ Data}

This set of three independent measurements (each of which have different systematics and inputs) offers a very powerful constraint on the neutrino backgrounds in antineutrino mode (Table 3.3).

\begin{tabular}{|l|c|c|}
\hline Source of Measurement & WS uncertainty & resultant error on $\bar{\nu}$ cross sections \\
\hline \hline $\mathrm{CC} \mathrm{QE} \cos \theta_{\mu}$ distribution & $7 \%$ & $2 \%$ \\
\hline $\mathrm{CC} 1 \pi^{+}$event sample & $15 \%$ & $5 \%$ \\
\hline muon lifetimes & $30 \%$ & $9 \%$ \\
\hline
\end{tabular}

Table 3.3: Wrong-sign extraction uncertainties as obtained from various independent sources in the $\bar{\nu}$ data. The resultant systematic uncertainty on $\bar{\nu}$ cross section measurements is obtained by assuming that wrong-signs comprise $30 \%$ of the total events. 
The resulting wrong-sign systematic on any antineutrino cross section measurement is at the $2 \%$ level, which is remarkable for a detector which does not possess event-by-event sign selection. Given this redundant approach, the wrong-sign contamination should not be considered prohibitive to producing meaningful antineutrino cross section and oscillation measurements at MiniBooNE. These techniques may also be useful for other experiments without magnetized detectors which have

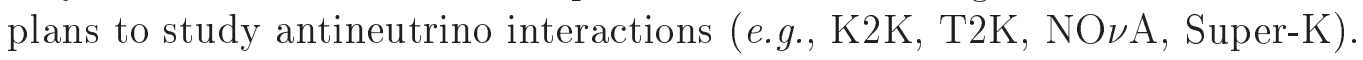

\subsubsection{Quasi-Elastic Scattering $\left(\bar{\nu}_{\mu} p \rightarrow \mu^{+} n\right)$}

Only a handful of experimental measurements exist on antineutrino quasi-elastic scattering cross sections (Figure 3.3 and Table 3.4). All come from bubble chamber measurements that were made on a variety of target materials with limited statistics. Note, in particular, that there is a complete lack of experimental data in MiniBooNE's energy range, below $1 \mathrm{GeV}$.
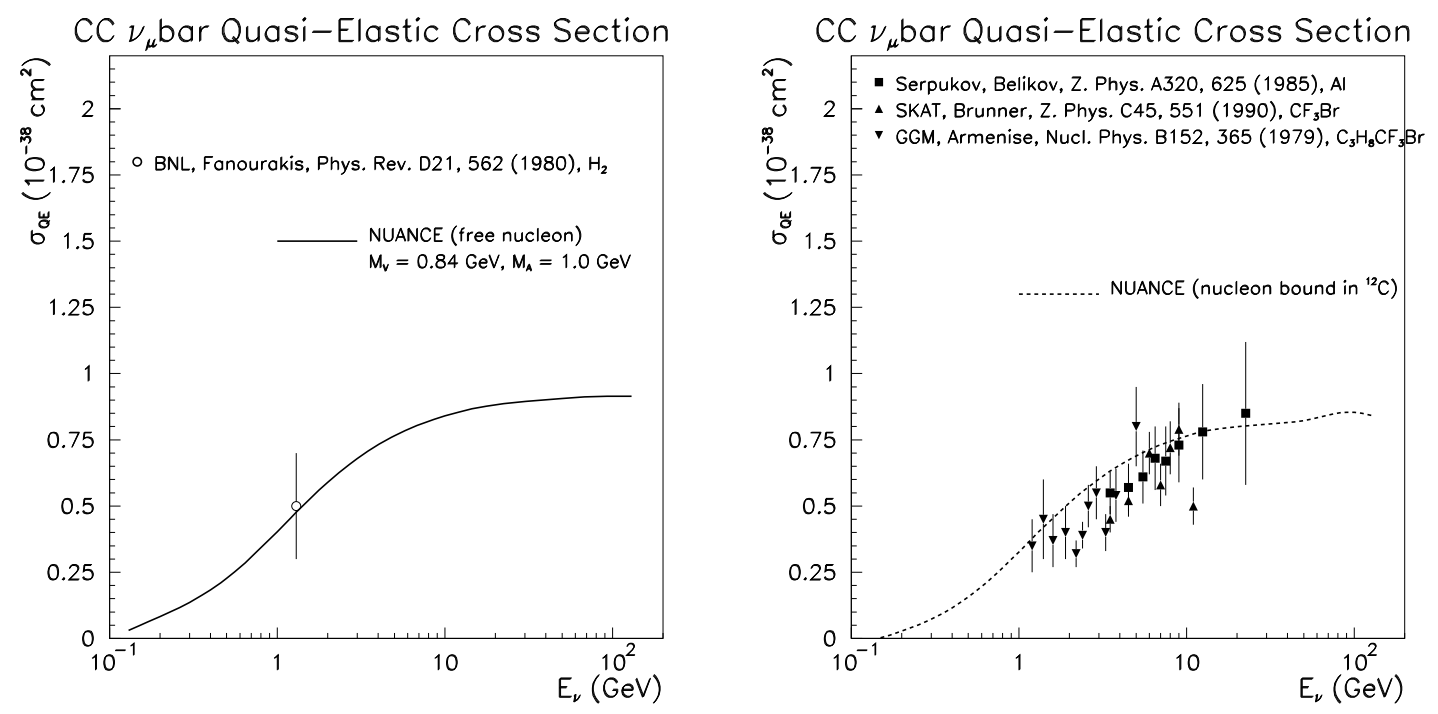

Figure 3.3: Existing experimental measurements of antineutrino QE scattering cross sections $\left(\bar{\nu}_{\mu} p \rightarrow \mu^{+} n\right)$ off light (left) and heavy (right) targets. The curves indicate the predictions from the NUANCE Monte Carlo generator [6].

\begin{tabular}{|l|c|c|c|c|}
\hline$\left.<E_{\bar{\nu}}\right\rangle$ & Experiment & target & date & $\# \bar{\nu}_{\mu}$ QE events \\
\hline \hline $2 \mathrm{GeV}$ & Gargamelle [11] & $C_{3} H_{8} C F_{3} B r$ & 1979 & 766 \\
\hline $1.3 \mathrm{GeV}$ & BNL [12] & $H_{2}$ & 1980 & 13 \\
\hline $16 \mathrm{GeV}$ & FNAL [13] & $\mathrm{NeH}_{2}$ & 1984 & 405 \\
\hline $6-7 \mathrm{GeV}$ & SKAT [14] & $\mathrm{CF}_{3} \mathrm{Br}$ & 1988 & 92 \\
\hline $9 \mathrm{GeV}$ & SKAT [15] & $C F_{3} B r$ & 1990 & 159 \\
\hline $5-7 \mathrm{GeV}$ & SKAT [16] & $C F_{3} B r$ & 1992 & 256 \\
\hline \hline
\end{tabular}

Table 3.4: Chronological listing of previous $\bar{\nu}_{\mu} Q E$ cross section measurements. 
To ensure the robustness of future $\mathrm{CP}$ violation measurements in the neutrino sector, one would prefer to avoid relying heavily on Monte Carlo predictions and extrapolations into regions where no data exist. MiniBooNE can serve an important role in this regard by making the first high statistics antineutrino QE measurement below $1 \mathrm{GeV}$. This section describes the feasibility of such a measurement.

MiniBooNE expects approximately 40,000 QE interactions in antineutrino mode with $2 \times 10^{20}$ POT before cuts (to be compared to 766 events from the next most sensitive measurement). Applying the same QE event selection criteria as in our neutrino data [17], yields a sample of $\sim 19,000$ events, $75 \%$ of which are pure QE interactions $\left(\nu_{\mu}+\bar{\nu}_{\mu} \mathrm{QE}\right)$. Roughly $23 \%$ of the events are neutrino wrong-sign interactions and $\sim 18 \%$ are non-QE $\bar{\nu}_{\mu}$ background events (Table 3.5).

\begin{tabular}{|c|c|}
\hline Event type & \# events passing CC QE selection \\
\hline \hline $\mathrm{CC} \mathrm{QE} \bar{\nu}_{\mu}(\mathrm{RS})$ & 10,893 \\
\hline $\mathrm{CC} \mathrm{QE} \nu_{\mu}(\mathrm{WS})$ & 3,332 \\
\hline $1 \pi \bar{\nu}_{\mu}$ backgrounds & 3,341 \\
\hline $1 \pi \nu_{\mu}$ backgrounds & 1,032 \\
\hline QE $\bar{\nu}_{\mu}$ hyperon production & 329 \\
\hline \hline total & 18,927 \\
\hline
\end{tabular}

Table 3.5: Events passing the CC QE event selection in $\bar{\nu}$ mode with $2 \times 10^{20}$ POT.

Assuming the wrong-sign constraint from Section 3.1.2 along with conservative errors on the incoming neutrino flux, the background contributions, and event detection together imply that MiniBooNE can measure the antineutrino QE cross section to better than $20 \%$ with $2 \times 10^{20}$ POT. Hence, even with one year of running, MiniBooNE can obtain an order of magnitude more $\bar{\nu}_{\mu}$ QE events than previous experiments, and be the first to measure the antineutrino QE cross section in this energy range.

\subsubsection{NC $\pi^{0}$ Production $\left(\bar{\nu}_{\mu} N \rightarrow \bar{\nu}_{\mu} N \pi^{0}\right)$}

To date, there is only one published measurement of the absolute rate of antineutrino NC $\pi^{0}$ production, the single largest background to future $\bar{\nu}_{\mu} \rightarrow \bar{\nu}_{e}$ oscillation searches; this measurement was reported with $25 \%$ uncertainty at $2 \mathrm{GeV}$ [18]. In addition, recent results from MiniBooNE [19] and K2K [20] have renewed interest in the relative rate of coherent versus resonant $\mathrm{NC} \pi^{0}$ production. Current theoretical models on coherent $\pi^{0}$ cross sections at low energy can vary by up to an order of magnitude in their predictions [21, 22]. Antineutrino scattering is uniquely suited to improve upon these constraints given that resonant contributions decrease, while coherent production remains the same [21,22]. This effectively amplifies the coherent contribution. Because coherently produced pions are emitted in the very forward direction, the relative resonant to coherent rate is revealed in the observed pion angular distributions. Figure 3.4 contrasts these distributions for neutrino and antineutrino interactions. In the neutrino case, the fraction of coherent $1 \pi^{0}$ events is half that in antineutrino mode. The coherent contribution is far more dramatic in the antineutrino data, thereby allowing a better measurement of the coherent $\mathrm{NC} \pi^{0}$ cross 

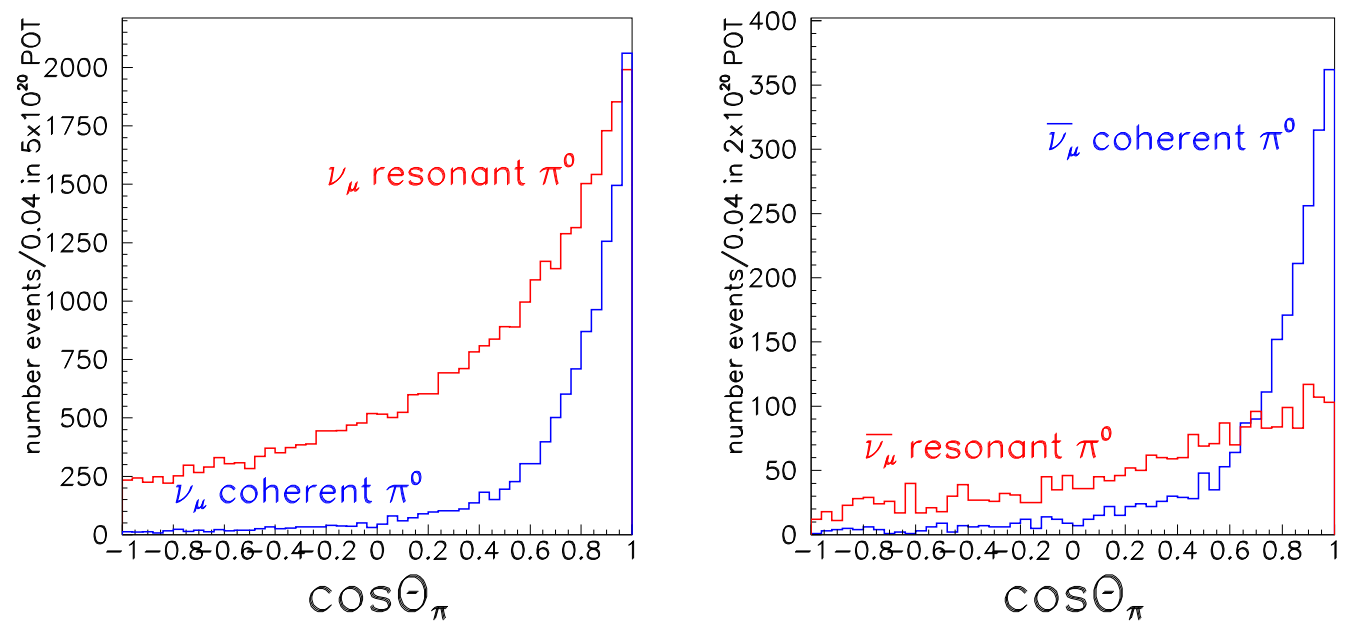

Figure 3.4: Generated $\pi^{0}$ angular distributions for $N C \nu$ (left) and $\bar{\nu}$ (right) scattering [6]. This is the angle of the outgoing $\pi^{0}$ in the lab with respect to the $\nu$ direction.

section.

Using the same cuts as in neutrino mode with no further optimization results in a clean sample of antineutrino NC $\pi^{0}$ events with a similar event purity and efficiency [19]. After this selection, we expect 1,650 $\bar{\nu}_{\mu}$ resonant $\mathrm{NC} \pi^{0}$ events and 1,640 $\bar{\nu}_{\mu}$ coherent NC $\pi^{0}$ events assuming an exposure of $2 \times 10^{20}$ POT. Figure 3.5 shows that the amplification of the coherent scattering component clearly persists even after reconstruction and event selection. Also displayed in the figure is the irreducible expected background of roughly $1,000 \nu_{\mu} \mathrm{NC} \pi^{0}$ events. Their relative contribution will be well predicted given the constraints on the wrong-sign content in the beam as described in Section 3.1.2 and the measurement of the $\nu_{\mu} \mathrm{NC} \pi^{0}$ cross section from MiniBooNE neutrino data.

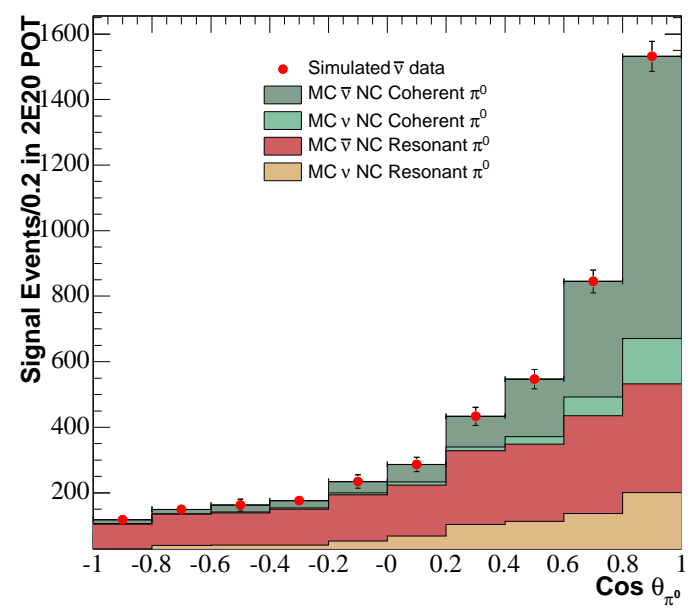

Figure 3.5: Reconstructed $\pi^{0}$ angular distributions for events passing the $N C \pi^{0}$ event selection in $\bar{\nu}$ mode. Shown are the individual contributions from both $\bar{\nu}_{\mu}$ and $\nu_{\mu}$ resonant and coherent $N C 1 \pi^{0}$ production for this mode of running. The errors on the simulated $\bar{\nu}$ data include both statistical and fit uncertainties. 
Since there are very little existing data on antineutrino NC $1 \pi^{0}$ production cross sections, measurement of this rate in antineutrino running at MiniBooNE would be of great value to the neutrino community and in particular to experiments proposing to look for $\bar{\nu}_{\mu} \rightarrow \bar{\nu}_{e}$ oscillations. In addition, we gain further insight on coherent $\pi^{0}$ production, which is itself of intrinsic interest. When the coherence length is on the order of or greater than the ${ }^{12} \mathrm{C}$ nucleus, the cross section is related by $\mathrm{PCAC}{ }^{1}$ to the elastic scattering cross section of $\pi$ 's from ${ }^{12} \mathrm{C}$. The verification of this effect in MiniBooNE would be the first instance of the observation of shadowing, in reactions other than DIS, using an electroweak probe.

\subsubsection{Resonant Single $\pi^{-}$Production $\left(\bar{\nu}_{\mu} N \rightarrow \mu^{+} N \pi^{-}\right)$}

In addition to the $\mathrm{CC} \mathrm{QE}$ and $\mathrm{NC} 1 \pi^{0}$ cross section measurements that can be made in antineutrino running at MiniBooNE, further exploration of CC resonant pion production rates is equally valuable. Because of the differing isospin content of the final states, the resonances produced in neutrino and antineutrino scattering are distinct (Table 3.6).

\begin{tabular}{|c|c|c|}
\hline neutrino type & CC $1 \pi$ channel & dominant resonance \\
\hline$\nu$ & $\nu_{\mu} p \rightarrow \mu^{-} p \pi^{+}$ & $\Delta^{++}$ \\
$\nu$ & $\nu_{\mu} n \rightarrow \mu^{-} p \pi^{0}$ & $\Delta^{+}$ \\
$\nu$ & $\nu_{\mu} n \rightarrow \mu^{-} n \pi^{+}$ & $\Delta^{+}$ \\
\hline $\bar{\nu}$ & $\bar{\nu}_{\mu} n \rightarrow \mu^{+} n \pi^{-}$ & $\Delta^{-}$ \\
$\bar{\nu}$ & $\bar{\nu}_{\mu} p \rightarrow \mu^{+} n \pi^{0}$ & $\Delta^{0}$ \\
$\bar{\nu}$ & $\bar{\nu}_{\mu} p \rightarrow \mu^{+} p \pi^{-}$ & $\Delta^{0}$ \\
\hline
\end{tabular}

Table 3.6: CC resonant single pion production modes for neutrino and antineutrino scattering. The dominant contributing baryonic resonance is indicated in each case.

Measuring resonant production in antineutrino data allows one to probe the various resonant components. The reaction $\bar{\nu}_{\mu} p \rightarrow \mu^{+} p \pi^{-}$holds particular interest because it is much more sensitive to the effects of resonances beyond the $\Delta(1232)$, interference between resonances, and non-resonant components. These effects are weakly constrained by limited antineutrino statistics from decades-old bubble chamber experiments (Table 3.7).

\begin{tabular}{|l|c|c|c|c|}
\hline$\left.<E_{\bar{\nu}}\right\rangle$ & Experiment & target & date & $\# \bar{\nu}_{\mu} p \rightarrow \mu^{+} p \pi^{-}$events \\
\hline \hline $1.5 \mathrm{GeV}$ & Gargamelle [24] & $C_{3} H_{8} C F_{3} B r$ & 1979 & 282 \\
\hline $5-70 \mathrm{GeV}$ & FNAL [25] & $H_{2}$ & 1980 & 247 \\
\hline $5-200 \mathrm{GeV}$ & BEBC [26] & $D_{2}$ & 1983 & 300 \\
\hline $25 \mathrm{GeV}$ & BEBC [27] & $H_{2}$ & 1986 & 375 \\
\hline $7 \mathrm{GeV}$ & SKAT [28] & $C F_{3} B r$ & 1989 & 120 \\
\hline
\end{tabular}

Table 3.7: Listing of previous measurements of $C C$ resonant $\bar{\nu}_{\mu} p \rightarrow \mu^{+} p \pi^{-}$events.

\footnotetext{
${ }^{1}$ Partially Conserved Axial Current [23]
} 
At MiniBooNE, roughly 7,000 resonant $\mathrm{CC} 1 \pi^{-}$are expected with $2 \times 10^{20}$ POT before cuts. Although most of the emitted $\pi^{-}$'s will be captured by carbon nuclei, such events still produce a signature: two Cerenkov rings (one each from the $\mu^{+}$and $\pi^{-}$) and one Michel electron from the muon decay. While promising, the selection efficiency and purity of such events is unknown at this time. Further investigation is currently underway.

\subsection{Oscillation Searches}

In addition to cross section advancements that can be made in one year of antineutrino running at MiniBooNE, several important oscillation scenarios can also be explored. These include $\bar{\nu}_{\mu}$ disappearance and $\bar{\nu}_{\mu} \rightarrow \bar{\nu}_{e}$ appeareance searches.

\subsection{1 $\quad \bar{\nu}_{\mu}$ Disappearance}

In sterile neutrino oscillation models, the rate of $\stackrel{(-)}{\nu_{\mu}}$ disappearance can be significantly larger than $\stackrel{(-)}{\nu_{\mu}} \rightarrow \stackrel{(-)}{\nu_{e}}$ appearance. Therefore, this search has the potential to provide information on additional mixing parameters as well as confirmation of the LSND signal. A disappearance search in antineutrino mode also provides a powerful test of CPT invariance. While CP violation can only be observed in an appearance experiment - by observing an asymmetry between the appearance rates in neutrinos and antineutrinos - the appearance mode is unable to distinguish if the asymmetry is the result of $\mathrm{CP}$ or CPT violation. As a result, one needs to additionally search for an asymmetry in a disappearance experiment. Moreover, the potential for a larger disappearance rate means that a disappearance asymmetry may be observable even if an appearance asymmetry is not.

The MiniBooNE disappearance sensitivity is estimated using the same event selection and systematic errors in neutrino and antineutrino running modes. The event selection results in an $80 \%$ pure CC QE sample with $50 \%$ efficiency within the fiducial volume $[1,17]$. This event selection reflects the current status and will likely improve. The systematic errors in the oscillation fit are dominated by uncertainties on the shape of the neutrino energy distribution, assumed to be 5\%. These preliminary systematic errors represent the eventual goals of the $\nu_{\mu}$ disappearance analysis.

The resultant MiniBooNE $90 \%$ confidence level $\bar{\nu}_{\mu} \rightarrow \bar{\nu}_{x}$ sensitivity, for $2 \times 10^{20}$ POT, is shown shown in Figure 3.6 under both CPT conserving (Figure 3.6a) and CPT violating (Figure 3.6b) assumptions. In the CPT conserving case, both $\nu_{\mu}$ 's and $\bar{\nu}_{\mu}$ 's in the antineutrino data are assumed to oscillate, whereas in the CPT violating case, only $\bar{\nu}_{\mu}$ 's are assumed to oscillate. For comparison, the $\nu_{\mu}$ disappearance sensitivity, expected by the end of FY2005, is shown in Figure 3.6a. Only a modest improvement in sensitivity is expected with increased statistics because both the $\nu_{\mu}$ and $\bar{\nu}_{\mu}$ disappearance analyses are systematics limited.

The strongest existing limit on $\nu_{\mu}$ disappearance comes from CDHS [29], as shown in Figure 3.6a. However, in the context of CPT violation, this limit does not apply to the $\bar{\nu}_{\mu}$ disappearance search. In this case, the best $\bar{\nu}_{\mu}$ disappearance limit comes 

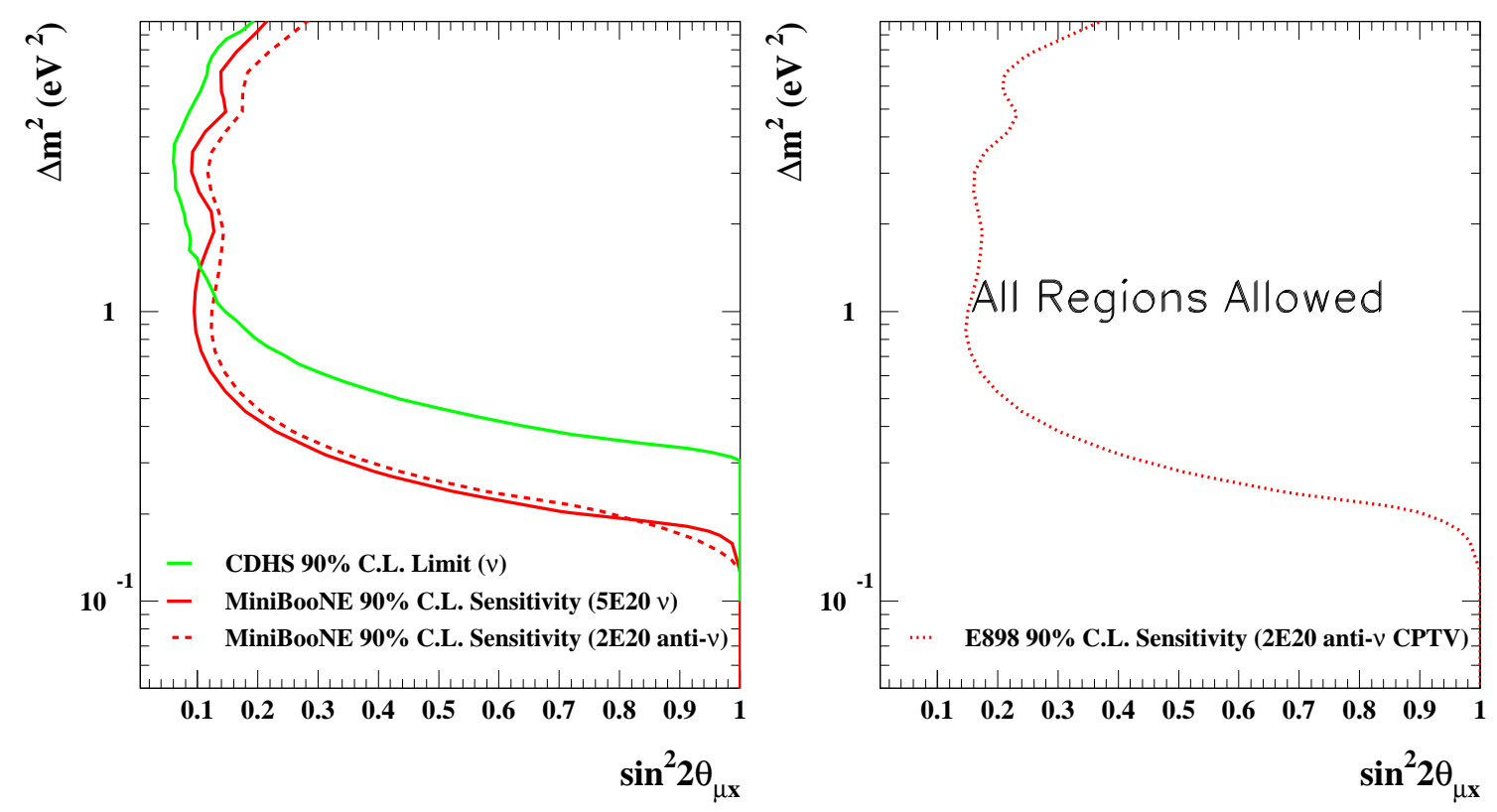

Figure 3.6: The MiniBooNE 90\% confidence level sensitivity under a) CPT conserving and b) CPT violating assumptions. In the CPT conserving case (left), the projected MiniBooNE sensitivity is shown for both $\nu_{\mu} \rightarrow \nu_{x}$ (with $5 \times 10^{20} P O T$ ) and $\bar{\nu}_{\mu} \rightarrow \bar{\nu}_{x}$ (with $2 \times 10^{20}$ POT) oscillations along with the $90 \%$ confidence level limit from CDHS [29]. In the CPT violating case (right), only the MiniBooNE $\bar{\nu}_{\mu} \rightarrow \bar{\nu}_{x}$ sensitivity is shown. In this instance, the existing experimental limits [30] are all above $10 \mathrm{eV}^{2}$ and hence the entire region shown on the righthand plot is currently allowed.

from CCFR [30], which is much less restrictive and off the scale of Figure 3.6b. As shown, the MiniBooNE $\bar{\nu}_{\mu}$ disappearance sensitivity in this case extends an order of magnitude lower than the current bounds of $\Delta m^{2} \sim 10 \mathrm{eV}^{2}$. In this way, by observing a different rate of $\nu_{\mu}$ versus $\bar{\nu}_{\mu}$ disappearance, MiniBooNE can be directly sensitive to $\mathrm{CPT}$ violation in the neutrino sector.

\subsection{2 $\bar{\nu}_{e}$ Appearance}

With the potential for $\mathrm{CP}(\mathrm{T})$-violation, testing the LSND oscillation hypothesis in the antineutrino mode in which it was originally observed is imperative. In order to make a definitive statement concerning LSND, it will therefore be extremely important for MiniBooNE to search for a signal with antineutrinos at a level sufficient to settle the issue. Although this physics requires more than $2 \times 10^{20} \mathrm{POT}$, we discuss our preliminary expectations for $\bar{\nu}_{e}$ appearance here in order to put FY2006 running into the context of a larger run plan.

Figure 3.7 shows an estimate of the MiniBooNE sensitivity to $\bar{\nu}_{\mu} \rightarrow \bar{\nu}_{e}$ oscillations in the scenario in which no $\nu_{\mu} \rightarrow \nu_{e}$ oscillations occur. In this calculation, a procedure similar to that employed in the 2003 Run Plan [1] was followed, with appropriate adaptations for antineutrinos. Most notably, while in antineutrino mode the number of neutrino interactions $\left(\bar{\nu}_{\mu}+\nu_{\mu}\right)$ per POT is about $29 \%$ of the number in neutrino 

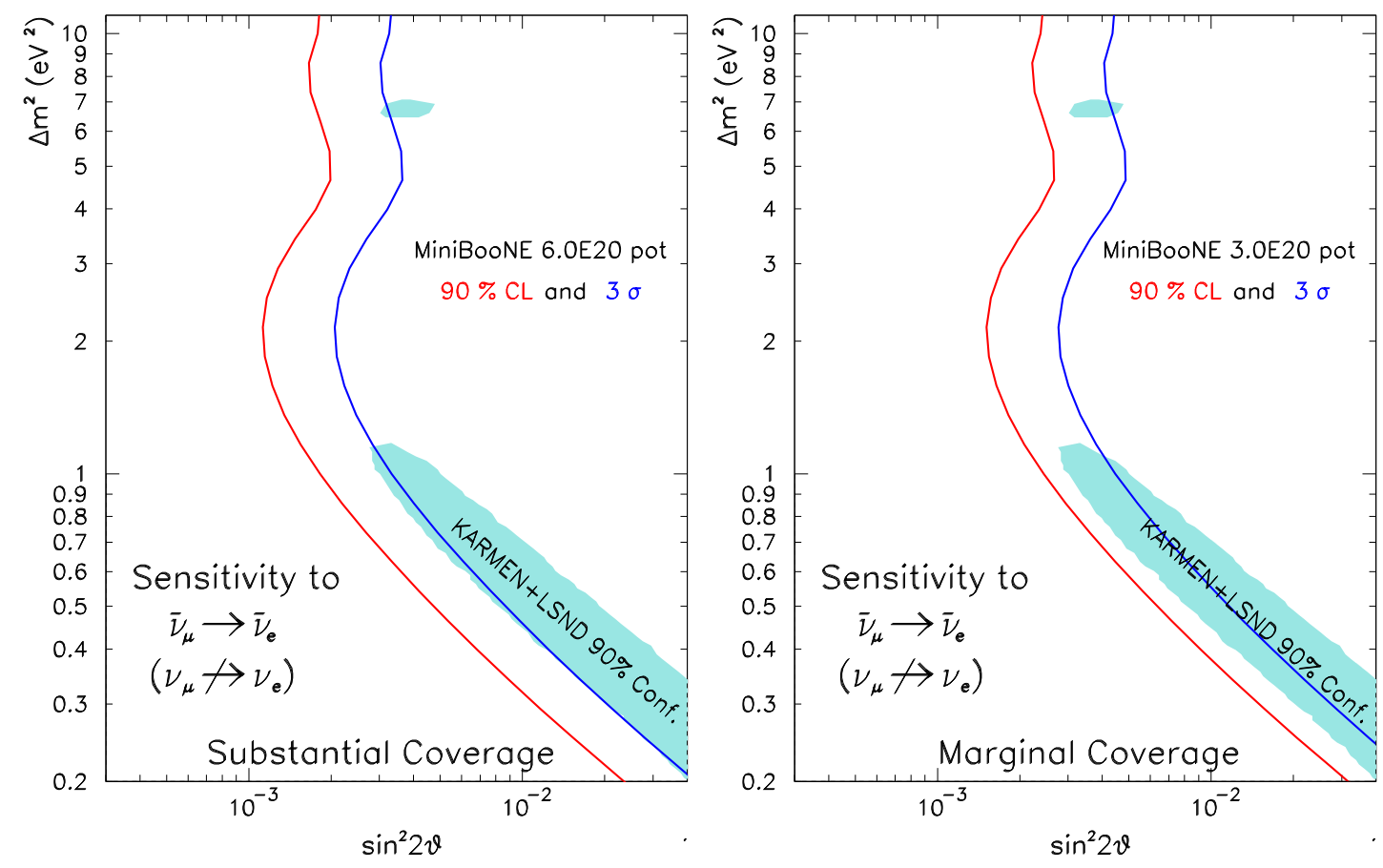

Figure 3.7: Sensitivity to $\bar{\nu}_{\mu} \rightarrow \bar{\nu}_{e}$ oscillations assuming no $\nu_{\mu} \rightarrow \nu_{e}$ oscillations using an energy spectrum fit. Shown in blue is the allowed $90 \%$ CL region from a joint analysis of the KARMEN and LSND $\bar{\nu}_{\mu} \rightarrow \bar{\nu}_{e}$ oscillations results [5]. Left (right) panel shows the result for $6 \times 10^{20}\left(3 \times 10^{20}\right)$ POT.

running mode, the intrinsic $\nu_{e}+\bar{\nu}_{e}$ background is a comparable fraction of the total event rate in both neutrino and antineutrino running. Here, we compare the sensitivity to the joint KARMEN-LSND region ( $\bar{\nu}$ only) [5] as opposed to the full LSND allowed region $(\nu+\bar{\nu})$. This smaller region provides the correct comparison given the difference in neutrino and antineutrino rates possible through $\mathrm{CP}$ violation.

Figure 3.7 shows two stops along the path toward decisive resolution of the LSND question in antineutrino mode. Delivery of $3 \times 10^{20}$ POT yields marginal (90\% CL) coverage of the KARMEN-LSND joint allowed region. $6 \times 10^{20}$ POT results in substantial $(3 \sigma)$ coverage. This is a statistics-limited search, thus further running will yield an even stronger statement. This should not be considered as a direct POT request at this time. As per the suggestion of the Director, we plan to make a formal request in 2005 for the protons on target needed for long-term running. 


\section{Chapter 4}

\section{Conclusions}

The LSND evidence for $\bar{\nu}_{\mu} \rightarrow \bar{\nu}_{e}$ appearance via neutrino oscillations is one of the most intriguing issues in particle physics today. If confirmed, the landscape of neutrino physics would be drastically altered, possibly by the addition of a new class of particles in the form of sterile neutrinos and new manifestations of CP violation. MiniBooNE is uniquely positioned to address the LSND result with data taken through FY2005 in neutrino mode. Analysis of the data is in progress, with the goal of producing a first result on $\nu_{\mu} \rightarrow \nu_{e}$ oscillations in the autumn of 2005. At that point, the collaboration has two options for how to proceed for the FY2006 run: continue in neutrino mode or switch to antineutrino running.

In the event of an inconclusive result that could stem from statistical fluctuations, MiniBooNE would continue in neutrino mode to strengthen the statistical significance of the existing result. The case for this program has been discussed in detail in the MiniBooNE Run Plan presented to the PAC in the Fall of 2003.

In the event of a strong confirmation of LSND, MiniBooNE may continue running in neutrino mode to better measure the oscillation parameters, or we may decide to reverse the polarity of the focusing horn and run with an antineutrino-enhanced beam. If LSND-like $\nu_{\mu} \rightarrow \nu_{e}$ oscillations are strongly excluded, MiniBooNE would choose to run with antineutrinos. In this document, we have outlined an important program of antineutrino physics that could be performed in this configuration with the $1-2 \times 10^{20}$ POT expected in FY2006. This includes dramatic improvements in our knowledge of $\bar{\nu}_{\mu}$ cross sections, and a search for $\bar{\nu}_{\mu}$ disappearance that would probe significantly further in oscillation parameter space than current limits. The cross section measurements are a necessary prelude to searches for CP violation in neutrino oscillations, including a $\bar{\nu}_{\mu} \rightarrow \bar{\nu}_{e}$ search at MiniBooNE that could be performed in a longer antineutrino run. A proposal for an extended antineutrino run would, in this case, be submitted to the PAC in 2005, and include a specific POT request.

We ask the PAC to endorse this strong physics program for FY2006 and recommend $1-2 \times 10^{20}$ POT be delivered to the MiniBooNE beam line. Furthermore, we ask the PAC to recommend that the laboratory continue to pursue a vigorous program to increase proton delivery. 


\section{Bibliography}

[1] A. A. Aguilar-Arevalo et al., "The MiniBooNE Run Plan" (2003).

[2] forthcoming DNP/DPF/DAP/DPB "Joint Study on the Future of Neutrino Physics", to appear on the DNP, DPF, DAP, and DPB websites (2004).

[3] M. Sorel, J M. Conrad, and M. Shaevitz, Phys. Rev. D70, 073004 (2004), hepph/0305255.

[4] V. Barger, M. Sorel, and K. Whisnant, in preparation.

[5] E. D. Church et al., Phys. Rev. D66, 013001 (2002), hep-ex/0203023.

[6] D. Casper, Nucl. Phys. Proc. Suppl. 112, 161 (2002), hep-ph/0208030.

[7] G. P. Zeller, proceedings of the 2nd Int'l Workshop on Neutrino-Nucleus Interactions, to be published in Nucl. Proc. Suppl., hep-ex/0312061.

[8] T. Suzuki et al., Phys. Rev. C35, 2122 (1987).

[9] S. Eidelman et al., Phys. Lett. B592, 33 (2004).

[10] M. O. Wascko, proceedings of the 2004 Meeting of the Division of Particles and Fields, to be published in Int. J. Mod. Phys. A.

[11] B. Armenise et al., Nucl. Phys. B152, 365 (1979).

[12] G. Fanourakis et al., Phys. Rev. D21, 562 (1980).

[13] A. E. Asratyan et al., Sov. J. Nucl. Phys. 39, 392 (1984).

[14] H. J. Grabosch et al., Sov. J. Nucl. Phys. 47, 1032 (1988).

[15] J. Brunner et al., Z. Phys. C45, 551 (1990).

[16] V. V. Ammosov et al., Sov. J. Nucl. Phys. 23, 283 (1992).

[17] J. Monroe, proceedings of the 39th Recontres du Moriond on Electroweak Interactions and Unified Theories, hep-ex/0406048.

[18] see footnote on page 235 in H. Faissner et al., Phys. Lett. 125B, 230 (1983).

[19] J. L. Raaf, proceedings of the 3rd Int'l Workshop on Neutrino-Nucleus Interactions, to be published in Nucl. Proc. Suppl., hep-ex/0408015. 
[20] S. Nakayama et al., submitted to Phys. Lett. B, hep-ex/0408134.

[21] D. Rein and L. M. Sehgal, Nucl. Phys. B223, 29 (1983).

[22] N. G. Kelkar et al., Phys. Rev. C55, 1964 (1997); J. Marteau et al., hepph/9906449; E.A. Paschos and A. V. Kartavtsev, hep-ph/0309148; B. Z. Kopeliovich, hep-ph/0409079.

[23] S. Adler, Phys. Rev. 135, B963 (1964).

[24] T. Bolognese et al., Phys. Lett. 81B, 393 (1979).

[25] S. J. Barish et al., Phys. Lett. 91B, 161 (1980).

[26] D. Allasia et al., Z. Phys. C20, 95 (1983).

[27] P. Allen et al., Nucl. Phys. B264, 221 (1986).

[28] H. J. Grabosch et al., Z. Phys. C41, 527 (1989).

[29] F. Dydak et al., Phys. Lett. B134, 281 (1984).

[30] I. E. Stockdale, C. Haber et al., Zeit. Phys. C27, 53 (1985); I. E. Stockdale, C. Haber et al., Phys. Rev. Lett. 52, 1384 (1984). 